MADPH-97-984

TUM-HEP-267/97

hep-ph/9702265

\title{
Model-independent Constraints on Topcolor from $R_{b}$
}

\author{
Gustavo Burdman \\ Department of Physics, University of Wisconsin, \\ Madison, WI 53706, USA \\ and \\ Dimitris Kominis|" \\ Institut für Theoretische Physik, Technische Universität München, \\ James-Franck-Straße, 85748 Garching, Germany
}

\begin{abstract}
We identify and compute the main corrections to $R_{b}$ in Topcolor theories. They are due to the top-pions, the pseudo-Goldstone bosons associated with the chiral symmetry breaking responsible for the top quark mass, and amount to $-(1-4) \%$, independent of the details of the Topcolor model. We show that the cancellation of this effect against the contributions from Topcolor vector and scalar resonances is unlikely, given the inherently distinct character of the two scales governing the potentially cancelling terms. The resulting value of $R_{b}$ leads to severe constraints on Topcolor theories.
\end{abstract}

*e-mail address: burdman@pheno.physics.wisc.edu

$\dagger$ †e-mail address: kominis@physik.tu-muenchen.de 


\section{Introduction}

The large value of the top-quark mass has led to speculation that the properties of the top quark may be fundamentally different from those of the other known fermions. In theories of top condensation, for example, a new strong gauge interaction (Topcolor), which couples to the $t$-quark and perhaps to other fermions as well, drives the formation of a $\langle\bar{t} t\rangle$ condensate and imparts a large dynamical mass to the top quark. In the process, the chiral symmetries associated with the strongly interacting fermions are spontaneously broken and at least three Goldstone bosons emerge. In the earliest incarnation of this idea [1], these Goldstone bosons were identified with the longitudinal components of the $W$ and $Z$ in an attempt to simultaneously resolve the puzzle of electroweak symmetry breaking. The Goldstone boson decay constant $f_{\pi}$ is approximately given in terms of the dynamical top mass $m_{t}$ and the scale $\Lambda$, at which Topcolor is assumed to break spontaneously to the Standard Model gauge group, by the Pagels-Stokar formula [0]

$$
f_{\pi}^{2} \approx \frac{N_{C}}{16 \pi^{2}} m_{t}^{2} \ln \frac{\Lambda^{2}}{m_{t}^{2}}
$$

where $N_{C}$ is the number of colors. If $f_{\pi}$ is to be identified with the weak scale $v_{w} \approx$ $175 \mathrm{GeV}$, then $\Lambda$ must be of the order of $10^{15} \mathrm{GeV}$. This, in turn, requires an extreme fine-tuning of the Topcolor gauge coupling in order to achieve a hierarchy of thirteen orders of magnitude between $m_{t}$ and $\Lambda$. Alternatively, one may wish to construct topcondensate theories which do not suffer from such unnatural hierarchies [3]. This means that the scale $\Lambda$ of the new gauge interactions should be brought down to a few $\mathrm{TeV}$ or so, depending on the amount of fine-tuning one is prepared to tolerate. From eq. (1) we then deduce that the Goldstone boson decay constant is $f_{\pi} \approx 60 \mathrm{GeV}$, a value insufficient to account for the observed $W$ and $Z$ masses. Consequently a separate mechanism, such as Technicolor (along with Extended Technicolor (ETC)) [4, must be invoked to supply the remainder of the weak gauge boson masses and give mass to the lighter fermions. The second major consequence of this approach is that the Goldstone bosons associated with the formation of the $\langle\bar{t} t\rangle$ condensate ("top-pions") are now physical degrees of freedom

Along with the light fermions, the top quark is expected to acquire itself a small hard mass of approximately $1 \mathrm{GeV}$ or so, from ETC interactions. This breaks the top chiral symmetries explicitly and turns the top-pions into massive pseudo-Goldstone bosons. A simple estimate gives [3]

$$
M_{\tilde{\pi}}^{2} \approx \frac{N_{C}}{8 \pi^{2}} \frac{m_{E T C} m_{t}}{f_{\pi}^{2}} \Lambda^{2} \gamma
$$

\footnotetext{
${ }^{1}$ More precisely, the linear combinations of top-pions and techni-pions which couple directly to the weak currents are absorbed via the Higgs mechanism, while the orthogonal linear combinations represent genuine physical states.
} 
where $\gamma$ is a radiative correction factor which can be rather large because of the strength of Topcolor interactions. For $m_{E T C} \sim 1 \mathrm{GeV}$, this yields $M_{\tilde{\pi}} \sim(100-300) \mathrm{GeV}$. Note that, because of its dependence on the parameter $m_{E T C}$, the top-pion mass represents an energy scale which is largely independent from, and much lower than the Topcolor scale $\Lambda$.

In this note we wish to point out that the presence of physical top-pions in the lowenergy spectrum is an inevitable feature of any Topcolor model that purports to avoid fine-tuning. The effects of top-pions on low-energy observables are governed by the scale $M_{\tilde{\pi}}$ (not the topcolor scale $\Lambda$ ), while the top-pion couplings to third generation quarks and to gauge bosons are to a large degree model-independent. Therefore, a study of toppion effects at low energies can be used to test the Topcolor idea as a whole, rather than constrain particular implementations of it.

With these comments in mind we investigate the impact of top-pions on $R_{b}$, the ratio of the $Z$ boson decay rate to $b \bar{b}$ pairs to its total hadronic width. This observable is expected to be sensitive to the Topcolor dynamics because of the non-trivial Topcolor interactions of the left-handed $b$-quark. We find that the top-pions lead to a substantial reduction of $R_{b}$ relative to the Standard Model prediction. While competing contributions are expected to arise in general [5], they are governed by the Topcolor scale $\Lambda$ and cannot compensate fully for the top-pion effects, despite the fact that they are formally leading in a $1 / N_{C}$ expansion. The crucial observation here is that, in addition to $1 / N_{C}$, the theory possesses another small parameter, namely $M_{\tilde{\pi}}^{2} / \Lambda^{2}$, which on symmetry grounds must vanish as $m_{E T C} \rightarrow 0$ independently of the model or the number of colors. In Sec. we derive the top-pion couplings to quarks in the effective Lagrangian approach. Their effect on $R_{b}$ is discussed in Sec. 3. Here we also take into account the possible effects of Topcolor vector mesons as well as those of additional scalar resonances present in specific models. We close with some concluding remarks in Sec. 1 .

\section{Low-energy Lagrangian}

The Topcolor gauge group is assumed to contain an $S U(3)_{1} \times S U(3)_{2}$ subgroup, which is spontaneously broken at an energy scale $\Lambda$ down to the $S U(3)$ of QCD. The doublet $\psi_{L}=\left(t_{L} b_{L}\right)^{T}$ as well as $t_{R}$ couple to $S U(3)_{1}$, which is the strongest of the two $S U(3)$ groups. The representation assignment of the remaining fermions is a model-dependent issue. However it should be made in such a way as to cancel gauge anomalies and ensure that no condensate of known quarks forms other than $\langle\bar{t} t\rangle$. Additional fermions may be enlisted in order for these requirements to be fulfilled. In this section we restrict attention only to the interactions of $\psi_{L}$ and $t_{R}$ which are common to all models. 
The quantitative study of this theory [3] begins with the simplifying assumption that, at energies below the scale $\Lambda$ the leading effects of the Topcolor dynamics are adequately described by a contact term

$$
\mathcal{L}=-\frac{2 \pi \kappa}{\Lambda^{2}}\left(\bar{\psi}_{L} \gamma_{\mu} \frac{\lambda^{A}}{2} \psi_{L}+\bar{t}_{R} \gamma_{\mu} \frac{\lambda^{A}}{2} t_{R}\right)^{2}
$$

which corresponds to the exchange of massive Topcolor gauge bosons. Here $\kappa$ is essentially the "fine-structure constant" of $S U(3)_{1}$ and $\lambda^{A}$ are the Gell-Mann matrices. Notice that the Lagrangian (3) is invariant under an $S U(2) \times U(1)$ chiral symmetry. By performing a Fierz transformation one can isolate the scalar channel, i.e. the contact interaction of Lorentz-scalar color-singlet bilinears, which is relevant for chiral symmetry breaking:

$$
\mathcal{L}_{\text {scalar }}=\frac{4 \pi \kappa}{\Lambda^{2}} \bar{\psi}_{L} t_{R} \bar{t}_{R} \psi_{L}
$$

Calculations with this effective lagrangian are usually carried out in the so-called NambuJona-Lasinio (NJL) approximation [6], which consists in resumming diagrams involving chains of fermion bubbles (but not loops of such chains). This approximation is justified in the limit where the number of colors $N_{C}$ becomes large.

Alternatively, one may introduce collective variables in the form of an auxiliary doublet field $\phi_{0}$ [7]. The Lagrangian (44) is equivalent to

$$
\mathcal{L}_{\text {scalar }}=-\lambda \bar{\psi}_{L} \phi_{0} t_{R}+\text { h.c. }-\Lambda^{2} \phi_{0}^{\dagger} \phi_{0}
$$

where $\lambda^{2}=4 \pi \kappa$. The renormalization group evolution of this Lagrangian to low energy scales generates a gauge-invariant kinetic term as well as self-interactions for $\phi_{0}$. In terms of the properly renormalized field $\phi$ the effective Lagrangian reads

$$
\mathcal{L}_{\text {eff }}=\left|D_{\mu} \phi\right|^{2}-\tilde{\lambda} \bar{\psi}_{L} \phi t_{R}+\text { h.c. }-V(\phi)
$$

where

$$
D_{\mu}=\partial_{\mu}-i g W_{\mu}^{a} \tau^{a}-i g^{\prime} \frac{Y}{2} B_{\mu}
$$

$\tau^{a}$ are $S U(2)$ generators and $Y=-1$ is the hypercharge of $\phi$. The mass-squared term in the potential $V(\phi)$ is negative leading to the spontaneous breakdown of the chiral symmetries to a $U(1)$ subgroup.

Note that the Lagrangian (6) is largely independent of the details of the above construction. From an effective field theory point of view, the expression (6) is the most 
general Lagrangian describing a $S U(2) \times U(1) \rightarrow U(1)$ symmetry breaking pattern and involving as the sole degrees of freedom (apart from the quarks and gauge bosons) an entire' scalar doublet $\phi$. The details of the potential $V(\phi)$ are irrelevant to our discussion, except that it is such as to cause $\phi$ to develop a nonzero vacuum expectation value, $f_{\pi}$. Since the top quark acquires the bulk of its mass through this mechanism, its Yukawa coupling to the scalar doublet is fixed: $\tilde{\lambda}=m_{t} / f_{\pi}$. The remaining couplings are determined by gauge invariance. As far as the effective theory is concerned, the value of $f_{\pi}$ is a free parameter. In the numerical estimates of the following sections we take $f_{\pi}=60 \mathrm{GeV}$ as suggested by the NJL approximation to the Pagels-Stokar formula (eq. (11)). The soft dependence on the generally unknown cutoff $\Lambda$ indicates that sharp deviations from this value are unlikely.

In terms of the component fields of the scalar doublet, defined by

$$
\phi=\left(\begin{array}{c}
f_{\pi}+\frac{1}{\sqrt{2}}\left(h+i \tilde{\pi}^{0}\right) \\
\tilde{\pi}^{-}
\end{array}\right)
$$

the Lagrangian (6) is given by

$$
\begin{aligned}
\mathcal{L}_{\text {eff }}= & \left|D_{\mu} \phi\right|^{2}-m_{t} \bar{t} t-\frac{m_{t}}{f_{\pi}}\left[\frac{1}{\sqrt{2}} \bar{t}_{L}\left(h+i \tilde{\pi}^{0}\right) t_{R}+\bar{b}_{L} \tilde{\pi}^{-} t_{R}+\text { h.c. }\right] \\
& \left.-\frac{1}{2} M_{h}^{2} h^{2}+\text { (scalar self }- \text { interactions }\right)
\end{aligned}
$$

where $M_{h}$ is the mass of the scalar state $h$. As explained above, the ETC interactions explicitly break the chiral symmetries and cause the states $\tilde{\pi}$ to acquire masses $M_{\tilde{\pi}^{ \pm}}, M_{\tilde{\pi}^{0}}$. These are not necessarily equal in general, because of the lack of an $S U(2)$ custodial symmetry. Finally, note that the mixing of $\tilde{\pi}^{ \pm, 0}$ with the Goldstone bosons of electroweak symmetry breaking introduces a factor of $\cos \theta$ to the fermion couplings of the physical top-pions, where $\sin \theta=f_{\pi} / v_{w}$, and generates a coupling between the top-pions and the right-handed $b$-quark. The latter is proportional to $m_{b}$ and will be neglected. Correcting for the former effect and keeping only the terms which are relevant to the calculation of the next section, we finally obtain

$$
\begin{aligned}
\mathcal{L}_{\mathrm{eff}}= & \left|D_{\mu} \phi\right|^{2}-m_{t} \bar{t} t-\frac{m_{t}}{f_{\pi}} \cos \theta\left[\frac{1}{\sqrt{2}} \bar{t} i \tilde{\pi}^{0} \gamma^{5} t+\bar{b}_{L} \tilde{\pi}^{-} t_{R}+\bar{t}_{R} \tilde{\pi}^{+} b_{L}\right] \\
& -M_{\tilde{\pi}^{ \pm}}^{2} \tilde{\pi}^{+} \tilde{\pi}^{-}-\frac{1}{2} M_{\tilde{\pi}^{0}}^{2} \tilde{\pi}^{02}+\ldots
\end{aligned}
$$

\footnotetext{
${ }^{2}$ The existence of a full scalar doublet at low energies is suggested by the NJL approximation and is a consequence of the necessity to keep the theory unitary up to the scale $\Lambda$. If one chose instead to write down a chiral effective Lagrangian keeping only the fermions and the Goldstone bosons, one would violate unitarity at a scale $\sim 4 \pi f_{\pi}<\Lambda$.
} 


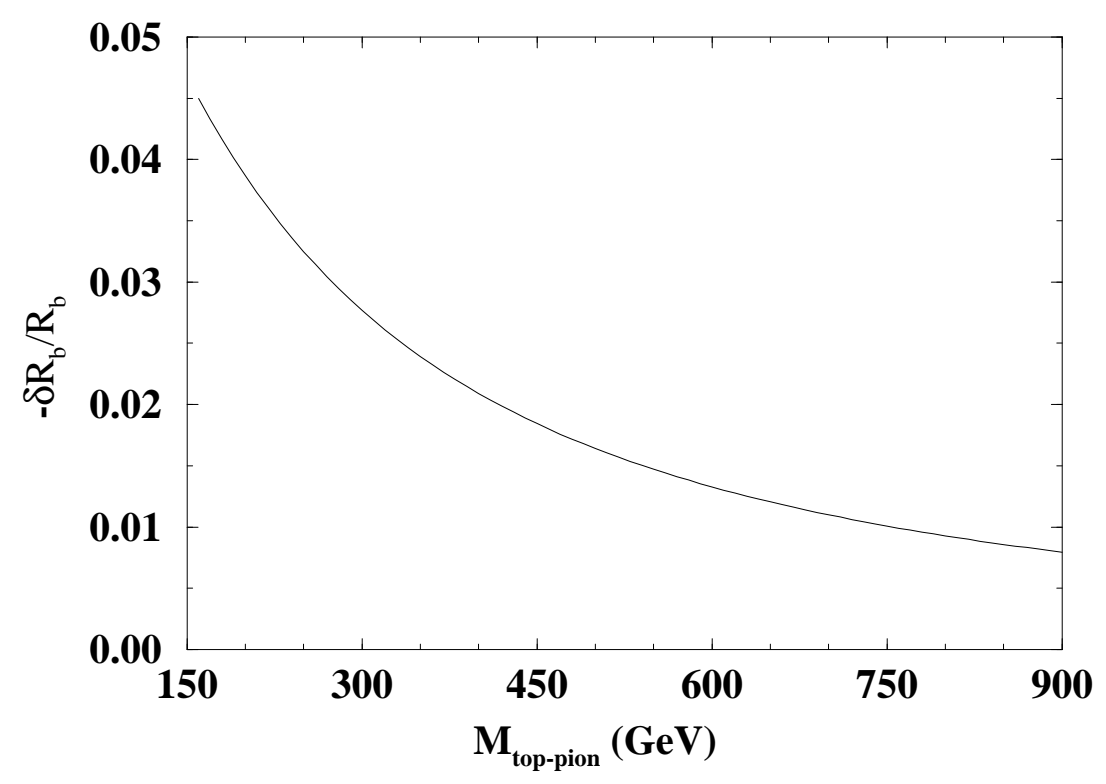

Figure 1: Fractional change in $R_{b}$ induced by the top-pions of a generic Topcolor model. Notice that the quantity plotted is the negative of the correction to $R_{b}$.

\section{$3 \quad R_{b}$ in Topcolor}

In Fig. 1 we plot the relative correction to $R_{b}$ due to top-pion exchange as a function of the mass of the charged top-pion. The calculation has been carried out to one loop with the Lagrangian (10) and follows closely the corresponding computation in the Two-HiggsDoublet model [8], as it can be seen in Fig. 2. The contributions of the neutral states $\tilde{\pi}^{0}$ and $h$ are suppressed at this level by a factor of order $\left(m_{b}^{2} / m_{t}^{2}\right)$ and are ignored. The arguments of the previous section imply that the only uncertainty in this result resides in the value of $f_{\pi}$. Of course, due to the strong couplings of the top-pions, higher-order corrections are expected to be substantial. We therefore regard our results only as rough estimates.

The collaborations at LEP have recently reported a measured value of $0.2178 \pm$ 0.0011 for $R_{b}$ [9] to be compared with the Standard Model prediction of 0.2158. This allows negative deviations relative to the Standard Model of only $0.6 \%$ at $3 \sigma$, and imposes very severe constraints on Topcolor models, unless there exist further contributions to $R_{b}$, which can naturally neutralize the top-pion effects. Candidates for such contributions include the exchange of Topcolor vector mesons or of additional light scalars which sometimes occur in specific models. We now turn to the study of these states and of their influence on $R_{b}$, adopting the NJL approximation as our calculational framework. 

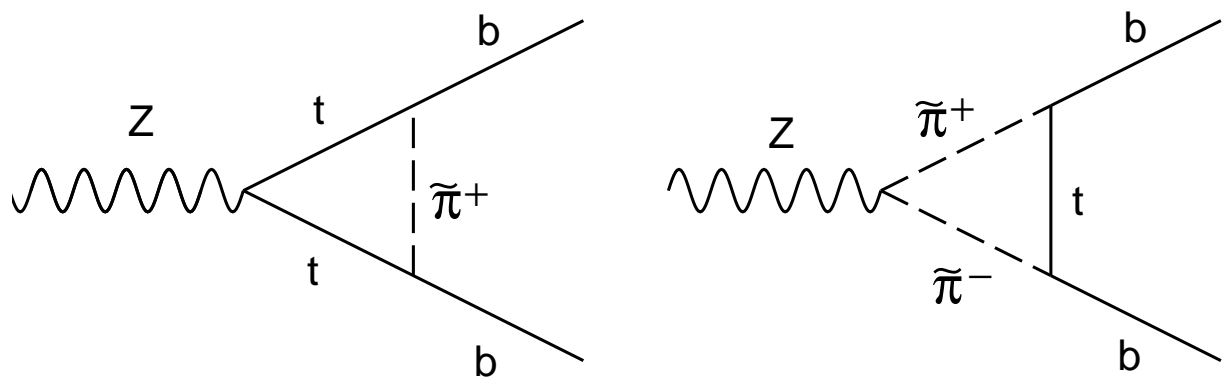

Figure 2: Corrections to the $Z \bar{b} b$ vertex due to top-pions. Not shown are the corresponding corrections to the fermion lines.

As mentioned in Section 2, a Topcolor model is specified when the anomaly-free assignment of all fermions is chosen so as to ensure that no SM fermion other than the top quark acquires a large dynamical mass. In particular, the $b$ quark will get a large mass if $b_{R}$ couples to the Topcolor interaction in the same way as $t_{R}$ does. This need for explicit isospin breaking has been addressed in various ways, all of which result in the presence of additional scalars in the spectrum. Here we show the effects of this model-dependent aspect of Topcolor on electroweak observables in the two scenarios described in [10].

First, let us consider the case where the condensation is tilted in the top direction by the presence of an extra $U(1)$ interaction embedded in the weak hypercharge group such that, at the scale $\Lambda, U(1)_{1} \times U(1)_{2} \rightarrow U(1)_{Y}$. Here the $U(1)_{1}$ is strongly coupled to the third generation fermions. The fermions have $U(1)_{1} \times U(1)_{2}$ assignments such that the couplings of the remnant $Z^{\prime}$ to fermions are analogous to those of the $Z$. Thus the effect of the $Z^{\prime}$ in the top channel adds to that of the Topcolor interaction, whereas it is repulsive in the $b$ quark channel. This defines a region in the coupling parameter space where only the coupling in the top channel is super-critical, leading to the breaking of the top chiral symmetry and to the scalar doublet in (8). In addition, in this region the effective interaction in the $b$ channel, although sub-critical, is strong enough to form rather deeply bound scalar states [1]. These make up the doublet

$$
\phi_{b}=\left(\begin{array}{c}
H^{+} \\
\frac{1}{\sqrt{2}}\left(H^{0}+i A^{0}\right)
\end{array}\right)
$$

Their masses can be estimated within the NJL approximation to be [11

$$
M_{H^{0}, A} \simeq(150-350) \mathrm{GeV}
$$

whereas the mass of the charged states is determined by the relation

$$
M_{H^{ \pm}}^{2}=M_{H^{0}, A}^{2}+2 m_{t}^{2} .
$$


The presence of this doublet adds the term

$$
-\tilde{\lambda} \bar{\psi}_{L} \phi_{b} b_{R}+\text { h.c. }
$$

to the effective Lagrangian (6), where as before $\tilde{\lambda}=m_{t} / f_{\pi}$. Therefore the charged scalars $H^{ \pm}$couple to $b_{R}$ with strength $\tilde{\lambda}$, whereas the neutrals $H^{0}, A^{0}$ have the same strong coupling to $\bar{b}_{L} b_{R}$. This results in additional contributions to $R_{b}$. The overall effect of the "b-pions" $H^{ \pm}$and $H^{0}, A^{0}$ is to further decrease $R_{b}$ by a small amount, making the deviation from the experimental result even larger. For instance, for $M_{H^{0}}=350 \mathrm{GeV}$ and $M_{\tilde{\pi}}=300 \mathrm{GeV}$, the value of $R_{b}$ is reduced by about $3.3 \%$. In the absence of the $b$-pions the effect is $\simeq 2.8 \%$. In these estimates we used the relation (13) for the $b$-pion masses, which however does not have to be taken literally. It is well-known [12] that the charged scalars in $\phi_{b}$ tend to reduce $R_{b}$ while the contribution from the neutrals is positive. The latter can only become dominant, however, if $M_{H^{0}, A} \ll M_{H^{ \pm}}$, in drastic departure from the NJL estimate (13). To reverse the top-pion effect one would further need $M_{H^{0}, A} \ll M_{\tilde{\pi}}$, which is counterintuitive. The relatively mild effect of the " $b$-pions" can be traced to the fact that the $Z$ boson decays predominantly into left-handed $b$-quarks, while in the limit of negligible $m_{b}$ the scalars $H^{ \pm}$couple exclusively to $b_{R}$.

One can further study the effects of vector and axial-vector mesons in Topcolor by essentially repeating the steps outlined at the beginning of Section 2 for the scalar doublet $\phi$. The methods employed have been extensively discussed in different contexts by various authors [13]. A vector or axial-vector resonance composed of $b$-quarks would contribute to $R_{b}$ by virtue of its mixing with the $Z$. In fact, taking into account this mixing amounts to summing all leading $1 / N_{C}$ contributions to the $Z b \bar{b}$ vertex. However, an estimate in the NJL approximation shows that these resonances are very loosely bound, that is, they have a mass of the order of the Topcolor scale $\Lambda$, and consequently the resummation hardly improves on the one-loop result of Ref. [5], according to which $R_{b}$ receives a positive correction of $\delta R_{b} / R_{b} \approx 2.5 \times 10^{-3}$ for $\Lambda=2 \mathrm{TeV}$ falling to $\delta R_{b} / R_{b} \approx 5.8 \times 10^{-4}$ for $\Lambda=5 \mathrm{TeV}$. This correction cannot compensate for the top-pion effects (cf. Fig. 1) which are governed by the - presumably much lower - scale $M_{\tilde{\pi}}$.

Another way to avoid the formation of a $\left\langle\bar{b}_{L} b_{R}\right\rangle$ condensate is to have the $b_{R}$ transforming as a $S U(3)_{1}$ singlet, so that it does not feel the Topcolor interaction. Following ref. [10], we notice that the cancelation of anomalies requires the introduction of a new set of fermion fields, $Q_{L, R}^{a}$, with $a=1, \ldots, N_{Q}$. There is a new interaction, $S U\left(N_{Q}\right)$. For instance for $N_{Q}=3$ the following assignments under $S U(3)_{Q} \times S U(3)_{1} \times S U(3)_{2}$ are anomaly-free:

$$
\begin{aligned}
(t, b)_{L}(c, s)_{L} & \simeq(1,3,1) \\
t_{R} & \simeq(1,3,1)
\end{aligned}
$$




$$
\begin{aligned}
Q_{R} & \simeq(3,3,1) \\
(u, d)_{L} & \simeq(1,1,3) \\
(u, d)_{R}(c, s)_{R} & \simeq(1,1,3) \\
b_{R} & \simeq(1,1,3) \\
Q_{L} & \simeq(3,1,3) .
\end{aligned}
$$

The fact that $(c, s)_{L}$ transforms as a triplet under $S U(3)_{1}$, in addition to $(t, b)_{L}$, implies that the chiral symmetry is now $S U(4)_{L} \times U(1)$. The breaking of this global symmetry by the Topcolor interactions now leads not only to the scalar doublet $\phi$ defined in Section 2 but also to the presence of an extra doublet of Goldstone bosons, the "charm-top-pions":

$$
\mathcal{C}=\left(\begin{array}{c}
\frac{1}{\sqrt{2}}\left(h_{c}+i \pi_{c}\right) \\
\pi_{c}^{-}
\end{array}\right)
$$

Although in principle massless, they acquire a mass generated by the same explicit chiral symmetry breaking responsible for $M_{\tilde{\pi}}$ and thus of similar value. Their coupling to the right-handed top quark is equal to that of the top-pions:

$$
-\tilde{\lambda}(\bar{c} \bar{s})_{L} \mathcal{C} t_{R}+\text { h.c. }
$$

This Topcolor model not only suffers from large deviations from the measured value of $R_{b}$, but in addition, the couplings in (16) imply large corrections to $R_{c}$. In Fig. 3 we plot the relative corrections to $R_{b}$ and to $R_{c}$ in this model as a function of the pseudo-Goldstone boson mass. Also shown is the $3 \sigma$ LEP result. Although the determination of $R_{c}$ is more uncertain, it can be seen that it can potentially pose an important additional constraint on these type of models. Note finally that the decay width of the $Z$ to light hadrons is also affected because $R_{s}$ is reduced by the same amount as $R_{b}$. In sum, this particular Topcolor model presents a lot of difficulties when confronted with observables at the $Z$ pole. In general, it is likely that other specific models will give rise to large deviations not only in $R_{b}$ but also in $R_{c}$ and the $Z$ hadronic width due to the ubiquitous presence of additional scalars in the spectrum.

\section{Conclusions}

We have seen that it is possible to constrain top condensation theories in general and Topcolor models in particular by concentrating on the effects due to the presence of toppions in the spectrum. Not only are these the minimal scalar content of any Topcolor model, but they also have a largely model-independent coupling to quarks, $\tilde{\lambda}=m_{t} / f_{\pi}$, allowing for rather general predictions. As it can be seen from (2), their masses are 


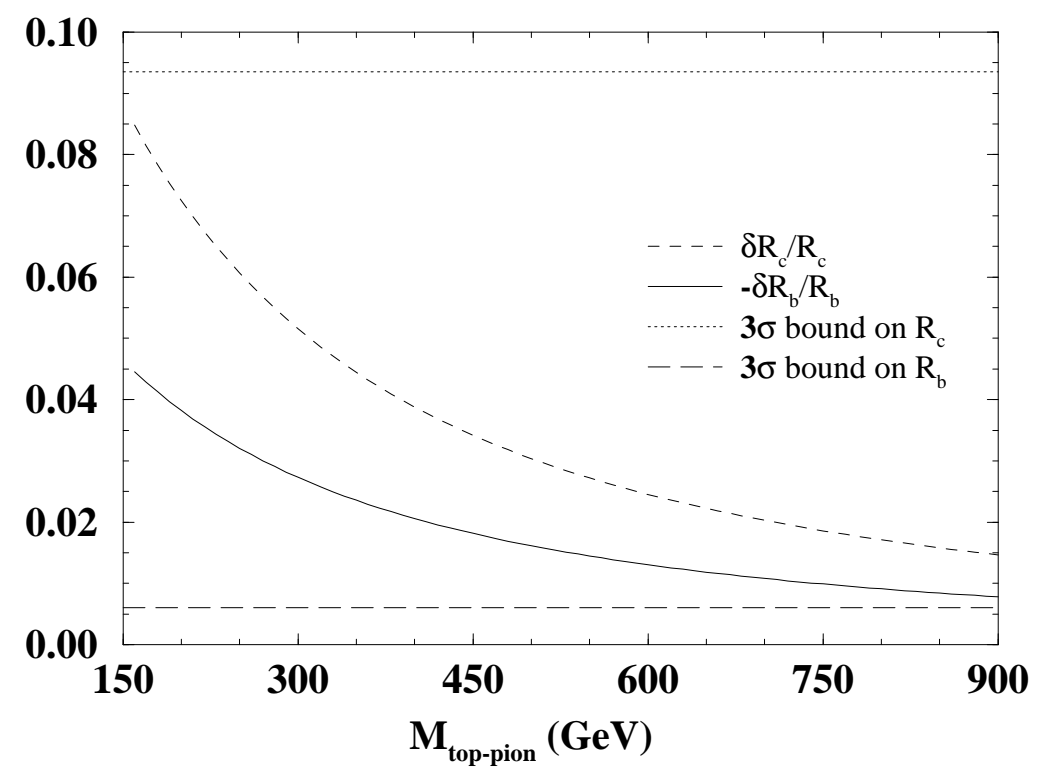

Figure 3: Fractional change in $R_{b}$ and $R_{c}$ in the Topcolor model with strongly coupled $(c, s)_{L}$ relative to the Standard Model value. The horizontal lines represent the experimental $3 \sigma$ upper bounds to these corrections. Note that, as in Fig. 1, the $R_{b}$ correction is negative.

expected to be well below the Topcolor scale $\Lambda$. This, together with the fact that their decay constant is expected to lie in the range $f_{\pi}=(50-70) \mathrm{GeV}$ in order to avoid fine-tuning, brings about the potential for strong effects, governed by the scale $M_{\tilde{\pi}}$ of a few hundred $\mathrm{GeV}$ rather than by the Topcolor scale itself. We computed the effect of toppions in $R_{b}$ and found it to be a large and negative shift with respect to the SM, as shown in Fig. 1. For typical values of $M_{\tilde{\pi}}$ this result is particularly constraining when compared to the experimental measurements at LEP [9], which at present point to a positive shift.

The effects of other Topcolor states were taken into account in order to investigate the possibility of cancellations. As noted in ref. [0], the contribution of the heavy topcolor gauge bosons, or "top-gluons", to $R_{b}$ is positive and small (less then $1 \%$ for realistic Topcolor scales). We considered the positive contributions of vector and axial-vector states and found that they are not enough to compensate for the negative shift due to the top-pions. We remark that, although the precise quantitative results depend on the approximation used, the contributions of these states are controlled by the scale $\Lambda$. Thus, a cancellation as large as the one required seems unlikely given the two different scales entering.

Attempts to involve some of the other standard quarks in the strong Topcolor interactions typically lead to additional light scalars which exacerbate the effect. We calculated the contributions to $R_{b}$ from these states in two specific realizations. In the model with 
an extra $U(1)$ we observe that the shift in $R_{b}$ is slightly more negative than in the sole presence of top-pions. The other model considered requires $(c s)_{L}$ to be a triplet under the strong $S U(3)_{1}$, i.e. to couple to the top-gluon. Here, in addition to the top-pions which modify the $Z b \bar{b}$ vertex in the same way as in the previous model, there is a new set of pseudo-Goldstone bosons, the charm-top-pions, which induce a very large positive shift to $R_{c}$. This also conflicts with experiment, but the discrepancy is milder than in the case of $R_{b}$. The situation is summarized in Fig. 3. There is also a large negative shift in $R_{s}$, of the same order as the one in $R_{b}$.

We conclude that a large and negative correction to $R_{b}$ is present in Topcolor models with minimal fermionic structure, and that there are no natural sources of cancellation, at least in the cases examined.

Our quantitative conclusions are of course valid only to the extent that the approximations we use are justified. Were $f_{\pi}$, for instance, allowed to float upwards by a factor of two relative to the Pagels-Stokar estimate, the correction to $R_{b}$ would be about 6.7 times smaller than the one presented in Fig. 1. Similarly, larger values of $M_{\tilde{\pi}}$ cannot be excluded given the uncertainty in the radiative correction factor $\gamma$ in eq. (2). The top-pion mass can also be enhanced by allowing larger values of $m_{E T C}$, but this option is rather unattractive.

A possible way out could in principle be provided by an extended fermionic content of the theory. If the top quark mixes with a new strongly interacting fermion of electric charge $2 / 3$, but possibly of "exotic" electroweak quantum numbers, then the correction to $R_{b}$ could be naturally smaller than in the case of no mixing. The challenge is to achieve this while avoiding the proliferation of pseudo-Goldstone bosons which will emerge from the breaking of a necessarily larger chiral symmetry group. Although the simplest models with additional fermions do not give rise to the desired symmetry breaking pattern, this remains perhaps an avenue to pursue further.

It should be remarked that other electroweak observables are also affected by the presence of light strongly interacting top-pions. For instance, the $\rho$ parameter receives a two-loop contribution from the latter in addition to the one from top-gluon exchange considered in ref. [14]. Any proposed mechanism introducing delicate cancellations in order to bring $R_{b}$ in line with experiment should also have a counterpart in the case of all other observables which are sensitive to the presence of pseudo-Goldstone bosons.

\section{Acknowledgements}

We thank Chris Hill for helpful discussions and a careful reading of the manuscript. DK thanks the Fermilab Theory Group for their warm hospitality during the initial stages of this work. His work was supported in part by the German DFG under contract number 
Li519/2-1. The work of GB was supported by the U.S. Department of Energy under

Grant No. DE-FG02-95ER40896 and the University of Wisconsin Research Committee with funds granted by the Wisconsin Alumni Research Foundation.

\section{References}

[1] Y. Nambu, in New Theories in Physics, Proceedings of the XI International Symposium on Elementary Particle Physics, Kazimierz, Poland, 1988, edited by Z. Adjuk, S. Pokorski and A. Trautmann (World Scientific, Singapore, 1989); Enrico Fermi Institute Report EFI 89-08 (unpublished); V. A. Miransky, M. Tanabashi and K. Yamawaki, Phys. Lett. 221B, (1989) 177; Mod. Phys. Lett. A4 (1989) 1043; W. A. Bardeen, C. T. Hill and M. Lindner, Phys. Rev. D41, (1990) 1647.

[2] H. Pagels and S. Stokar, Phys. Rev. D20 (1979) 2947.

[3] C. T. Hill, Phys. Lett. B345 (1995) 483.

[4] S. Weinberg, Phys. Rev. D19 (1979) 1277;

L. Susskind, Phys. Rev. D20 (1979) 2619;

S. Dimopoulos and L. Susskind, Nucl. Phys. B155 (1979) 237;

E. Eichten and K. Lane, Phys. Lett. B90 (1980) 125.

[5] C. T. Hill and X. Zhang, Phys. Rev. D51 (1995) 3563.

[6] Y. Nambu and G. Jona-Lasinio, Phys. Rev. 122 (1961) 345; 124 (1961) 246.

[7] T. Eguchi, Phys. Rev. D14 (1976) 2755.

[8] A. Denner, R. Guth, W. Hollik and J. H. Kühn, Z. Phys. C51 (1991) 695.

[9] A. Blondel, Plenary talk at the 28th International Conf. on High Energy Physics, Warsaw, July 1996.

[10] G. Buchalla, G. Burdman, C. T. Hill and D. Kominis, Phys. Rev. D53 (1996) 5185.

[11] D. Kominis, Phys. Lett. B358 (1995) 312.

[12] P. Bamert et al., Phys. Rev. D54 (1996) 4275.

[13] V. Bernard and U.-G. Meissner, Nucl. Phys. A489 (1988) 647; J. Bijnens, C. Bruno and E. de Rafael, Nucl. Phys. B390 (1993) 501; J. Bijnens, E. de Rafael and H. Zheng, Z. Phys. C62 (1994) 437; J. Bijnens, Phys. Rep. 265 (1996) 369.

[14] R. S. Chivukula, B. A. Dobrescu and J. Terning, Phys. Lett. B353 (1995) 289. 